Research Paper

\title{
Thirty-year trends in clinicopathologic characteristics and prognosis after gastrectomy for gastric cancer: A single institution in Northern China
}

\author{
Yu-en Tan, Peng-liang Wang, Song-cheng Yin, Chao Zhang, Wen-bin Hou, Hui-mian Xu ${ }^{凶}$ \\ Department of Surgical Oncology, First Affiliated Hospital of China Medical University, Shenyang, China. \\ $\square$ Corresponding author: Hui-Mian Xu, MD, PhD, Department of Surgical Oncology, the First Affiliated Hospital of China Medical University, Shenyang, \\ China. 155 Nanjing North Street, Heping District, Shenyang, China 110001, E-mail: huimianxucmu@126.com Tel: +86 13998303068 Fax: +86 24-22834060 \\ (c) The author(s). This is an open access article distributed under the terms of the Creative Commons Attribution License (https://creativecommons.org/licenses/by/4.0/). \\ See http:/ /ivyspring.com/terms for full terms and conditions.
}

Received: 2019.05.22; Accepted: 2019.10.26; Published: 2020.01.01

\begin{abstract}
Background: We integrated changes in the trends in clinicopathologic characteristics and postoperative prognosis in patients with gastric cancer Northern China over a 30-year period.

Methods: A retrospective analysis of patients undergoing gastric cancer resection and complete follow-up information from January 1981 to December 2010 in the first affiliated Hospital of China Medical University was carried out. We divided the patients into three consecutive periods.

Results: A total of 3,520 patients were included in this study. The proportion of lower tumors increased (from 58.8 to $66.9 \%$ ), while that of upper tumors decreased (from 21.3 to 13.4\%). The proportion of tumors $>5 \mathrm{~cm}$ decreased (from 58.6 to $41.1 \%$ ), but the increasing trend of poorly differentiated gastric cancer was obvious (from 60.1 to $75.7 \%$ ). The percentage of early gastric cancer increased from 10.0 to 15.5 during the study periods, and that of TNM stage IV cancer decreased from 38.6 to 28.1. In surgery treatment, the rate of radical resection increased to $92.1 \%$ in recent period, and the average number of retrieved lymph nodes increased. The 5-year survival rate gradually increased from $36.5 \%$ to $48.5 \%(p<0.001)$. The Multivariate analysis showed that age, tumor size, $\mathrm{T}$ stage, $\mathrm{N}$ stage, number of retrieved lymph nodes and resection type were independent prognostic factors for gastric cancer.

Conclusion: The patterns of clinicopathologic features for gastric cancer changed during the 30 -year period in North China. Overall survival (OS) could be increased by early detection of tumors and standard surgical treatment.
\end{abstract}

Key words: gastric cancer; time tendency; clinicopathologic characteristics; prognosis.

\section{Introduction}

Gastric cancer is the fifth most commonly diagnosed cancer and the third leading cause of cancer death (1). China has a high-incidence of gastric cancer, with about 400,000 new cases each year, and about 350,000 cases of gastric cancer, accounting for $40 \%$ of case globally $(2,3)$. At present, surgical resection is the most effective treatment for gastric cancer, but when distant metastasis occurs, chemotherapy is required.

There are many factors that affect the survival rate of patients with gastric cancer, including tumor grade, and histologic type. Large sample data reveal in detail the impact of clinicopathological factors on the prognosis of patients with gastric cancer $(4,5)$. Although controversial, it is of great significance to evaluate the efficacy of surgical treatment and carry out prospective clinical research to improve the comprehensive treatment of gastric cancer. In recent years, foreign researchers has become increasingly aware of the importance of changing treads in the 
clinicopathological factors for gastric cancer (6-9), but there are few similar reports in China (10).

Based on the collected data of gastric cancer patients in China, we assessed the time changes of the clinicopathologic features and postoperative outcome from 1981 to 2010, with the purpose of introducing the present situation and changes of gastric cancer in China.

\section{Materials and methods}

\section{Patient Source}

We obtained retrospective date for patients with gastric cancer treated surgically from January 1981 to December 2010 at the single center of the first affiliated Hospital of China Medical University. We included patients with pathologically diagnosed gastric adenocarcinoma with detailed medical records and complete pathological and follow-up data after surgery. Exclusion criteria were: (1) patients with neoadjuvant therapy; (2) patients who underwent non-tumor resection such as laparotomy and gastrojejunostomy; (3) patients with other gastric malignant tumors (such as gastric lymphoma and gastrointestinal stromal tumor) or postoperative recurrence of gastric cancer; (4) patients with other, simultaneous primary malignant tumors, such as colorectal, or breast cancer; and (5) patients who died from surgical complications during the perioperative period. Eventually, a total of 3520 gastric cancer patients were enrolled in our study.

We divided the patients into three chronological groups in order: 1981-1990 (period 1), 1991-2000 (period 2), and 2000-2010 (period 3). The detailed demographic and clinicopathological features of patients in the three groups are presented in Table $\mathbf{1}$. All the biopsies were performed by pathologists using standardized protocols.

The tumor sites in the stomach were uniformly classified as the upper, middle and lower. The histological types were well/moderately and poorly differentiated carcinoma. In accordance with the eighth edition of the TNM staging system of the American Joint Committee on Cancer/Union for International Cancer Control (AJCC/UICC) (11), the patients were divided into eight subgroups on the basis of their post-operative pathology.

All patients received standardized follow-up, based on telephone interviews or brief messages after discharge, every 3 months in the first 3 years, every 6 months in the fourth and fifth years, and every year thereafter until death or final follow-up (June 30, 2015).

\section{Statistical analyses}

We used SPSS version 20.0 for statistical analysis (SPSS Inc., Chicago, IL, USA). Differences in categorical variables were analyzed $\times 2$ test. KaplanMeier method was used to analyze the overall cumulative survival rate. Differences among the groups were depended on log rank test; A Cox regression model was used for multivariate analysis. Two-sided $\mathrm{P}<0.05$ was regarded as statistically significant.

Table 1. Comparison of clinicopathologic features in 3,520 patients with gastric adenocarcinoma after gastrectomy from 1981 to 2010.

\begin{tabular}{|c|c|c|c|c|}
\hline Factor & $\begin{array}{l}\text { Period } 1 \\
n=572, n(\%)\end{array}$ & $\begin{array}{l}\text { Period } 2 \\
\mathrm{n}=781, \mathrm{n}(\%)\end{array}$ & $\begin{array}{l}\text { Period } 3 \\
\mathrm{n}=2167, \mathrm{n}(\%)\end{array}$ & $\mathrm{P}$ valve \\
\hline Gender & & & & $<0.001$ \\
\hline Male & $463(80.9)$ & $561(71.8)$ & 1542 (71.1) & \\
\hline Female & 109 (19.1) & $220(28.2)$ & 625 (28.9) & \\
\hline Age & & & & $<0.001$ \\
\hline$\leq 60$ & 393 (68.7) & 401 (51.3) & 1198 (55.3) & \\
\hline$>60$ & $179(31.3)$ & $380(48.7)$ & 969 (44.7) & \\
\hline Tumor location & & & & $<0.001$ \\
\hline Upper & $122(21.3)$ & 139 (17.8) & $290(13.4)$ & \\
\hline Middle & $114(19.9)$ & $156(20.0)$ & 438 (19.7) & \\
\hline Lower & $336(58.8)$ & $486(62.2)$ & 1449 (66.9) & \\
\hline Histologic type & & & & $<0.001$ \\
\hline Well/moderately & $228(39.9)$ & $217(27.8)$ & $526(24.3)$ & \\
\hline Poorly & $344(60.1)$ & $564(72.2)$ & 1641 (75.7) & \\
\hline Tumor size & & & & $<0.001$ \\
\hline$\leq 5 \mathrm{~cm}$ & $237(41.4)$ & 395 (50.6) & 1270 (58.6) & \\
\hline$>5 \mathrm{~cm}$ & $335(58.6)$ & $386(49.4)$ & 897 (41.4) & \\
\hline T stage & & & & $<0.001$ \\
\hline $\mathrm{T} 1$ & $57(10.0)$ & $84(10.8)$ & 336 (15.5) & \\
\hline $\mathrm{T} 2$ & $84(14.7)$ & $112(14.3)$ & $298(13.8)$ & \\
\hline T3 & $152(26.6)$ & 293 (37.5) & 818 (37.7) & \\
\hline $\mathrm{T} 4$ & $279(48.8)$ & $292(37.4)$ & $715(33.0)$ & \\
\hline N stage & & & & $<0.001$ \\
\hline No & $184(32.2)$ & 244 (31.3) & 767 (35.4) & \\
\hline N1 & $119(20.8)$ & $196(25.1)$ & $353(16.3)$ & \\
\hline N2 & $143(25.0)$ & $158(20.2)$ & 406 (18.7) & \\
\hline N3 & $126(22.0)$ & $183(23.4)$ & $641(29.6)$ & \\
\hline TNM stage & & & & $<0.001$ \\
\hline IA & $49(8.6)$ & $61(7.8)$ & $285(13.2)$ & \\
\hline IB & $38(6.6)$ & $56(7.2)$ & $162(7.5)$ & \\
\hline IIA & $53(9.3)$ & $95(12.2)$ & $220(10.2)$ & \\
\hline IIB & 64 (11.2) & $98(12.5)$ & $235(10.8)$ & \\
\hline IIIA & 95 (16.7) & $121(15.5)$ & 305 (14.1) & \\
\hline IIIB & $41(7.2)$ & $65(8.3)$ & $218(10.1)$ & \\
\hline IIIC & $11(1.9)$ & $24(3.1)$ & $134(6.2)$ & \\
\hline IV & $221(38.6)$ & $261(33.4)$ & $608(28.1)$ & \\
\hline $\begin{array}{l}\text { NO. of retrieved lymph } \\
\text { node }\end{array}$ & & & & $<0.001$ \\
\hline$\leq 15$ & $351(61.4)$ & $436(55.8)$ & 396 (18.3) & \\
\hline$>15$ & $221(38.6)$ & $345(44.2)$ & 1771 (81.7) & \\
\hline Resection type & & & & $<0.001$ \\
\hline Radical & $437(76.4)$ & $647(82.8)$ & $1996(92.1)$ & \\
\hline Palliative & $135(23.6)$ & $134(17.2)$ & $171(7.9)$ & \\
\hline
\end{tabular}


A

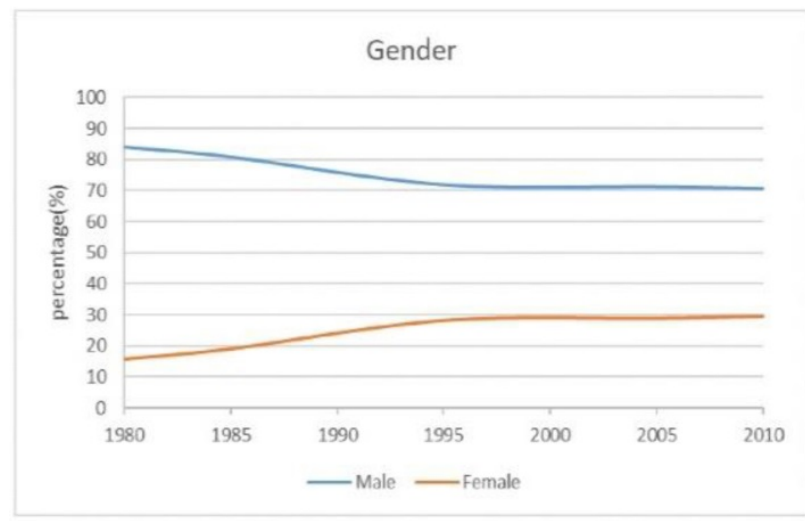

B

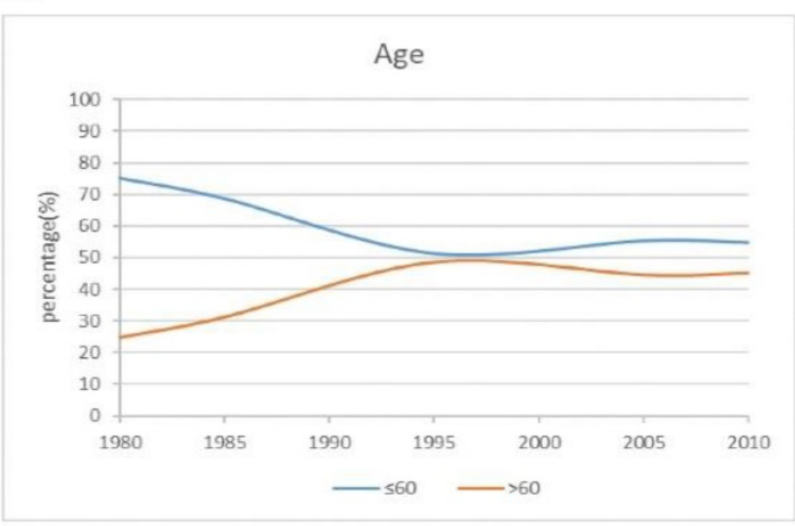

Figure 1. The curve of demographic characteristics changes over time for the patients.

\section{Results}

\section{Demographic characteristics}

A total of 3520 gastric cancer patients who met the above criteria were included in the study. The number of patients undergoing surgery increased from 581 in the period 1 to 2167 in the period 3 . There were 2566 men and 954 women, with a male to female ratio of 2.7: 1, and the proportion of male patients was lower than that in recent years. The mean age was 58.2 years, and the proportion of patients older than 60 years was greater in periods 2 and 3 than in period 1 (Figure 1).

\section{Pathological characteristics}

Most tumors were located in the lower stomach. The largest proportion of lower tumors gradually increased from $58.8 \%$ in period 1 to $66.9 \%$ in period 3 . On the contrary, upper tumors showed a slight downward trend. Changes in the histologic types of tumor in the three periods are shown in Figure 2. The proportion of well/moderately differentiated tumors decreased from $39.9 \%$ in period 1 to $24.3 \%$ in period 3 , and poorly differentiated tumors showed an upward tendency in the three periods. There was a gradual increase over time in tumors $>5 \mathrm{~cm}$ compared with tumors $<5 \mathrm{~cm}$. The proportion of patients with early gastric cancer (EGC) increased from $10 \%$ in period 1 to $15.5 \%$ in period 3 . There was no obvious trend in relation to $\mathrm{N}$ stage. Similar to $\mathrm{T}$ stage, the proportion of patients with stage IA curve of TNM stage showed an upward trend, reaching 13.2 $\%$ in 3 periods. The proportion of patients with stage IV cancer decreased from $38.6 \%$ in period 1 to $28.1 \%$ in period 3.

\section{Surgical treatment}

The number of lymph node metastases is one of the important indexes of clinical stage, and it can be used to evaluate surgical quality. The greater the number of lymph nodes dissected, the more favorable it is to analyze and evaluate the prognosis of patients. In this study, 15 lymph nodes were used as the breakpoint. The proportion of patients with dissection of $>15$ lymph nodes increased significantly from 38.6 $\%$ in period 1 to $81.7 \%$ in period 3 . The percentage of patients who underwent radical resection increased significantly, reaching $92.1 \%$ in period 3 (Figure 3).

\section{Prognosis}

To remove the effect of surgical mortality, patients who died 30 days after surgery were excluded. Median follow-up was 138.8 (54.7-431.7) months. The 5-year survival rates in periods 1 and 2 were $36.5 \%$ and $37.8 \%$ respectively, and increased significantly to $48.5 \%$ in the period $3(\mathrm{p}<0.001)$ (Figure 4).

\section{Univariate and multivariate analyses}

To establish the relationship between clinicopathological factors and prognosis of gastric cancer, we used the Cox regression model to analyze the patients. Univariate analysis showed that factors affecting prognosis of gastric cancer included age, tumor size, $\mathrm{T}$ stage, $\mathrm{N}$ stage, $\mathrm{M}$ stage, number of retrieved lymph nodes, surgical methods and time period. Sex, tumor location, histological type and other factors had no correlation with prognosis of gastric cancer. Multivariate analysis showed that age, tumor size, T-stage, $\mathrm{N}$ stage, number of retrieved lymph nodes and resection type were independent prognostic factors for gastric cancer (Table 2). 
Table 2. Univariate and multivariate analysis of risk factors in 3520 patients with gastric cancer.

\begin{tabular}{|c|c|c|c|c|c|c|c|c|}
\hline \multirow[b]{2}{*}{ Variable } & \multicolumn{4}{|c|}{ Univariate analysis } & \multicolumn{4}{|c|}{ Multivariate analysis } \\
\hline & SE & HR & $95 \% \mathrm{CI}$ & P valve & SE & HR & $95 \% \mathrm{CI}$ & P valve \\
\hline Gender & 0.046 & 0.990 & $0.905-1.084$ & .834 & - & - & - & - \\
\hline Age & 0.041 & 1.368 & $1.262-1.482$ & $<.001$ & 0.041 & 1.318 & $1.215-1.429$ & $<.001$ \\
\hline Tumor location & 0.027 & 0.988 & $0.936-1.042$ & .649 & - & - & - & - \\
\hline Histologic type & 0.033 & 0.951 & 0.891-1.015 & .133 & - & - & - & - \\
\hline Tumor size & 0.041 & 1.974 & $1.822-2.139$ & $<.001$ & $<.001$ & 1.100 & $1.008-1.200$ & $<.032$ \\
\hline T stage & 0.022 & 1.694 & $1.621-1.771$ & $<.001$ & 0.033 & 1.193 & $1.118-1.273$ & $<.001$ \\
\hline $\mathrm{N}$ stage & 0.018 & 1.625 & $1.569-1.682$ & $<.001$ & 0.027 & 1.365 & $1.294-1.440$ & $<.001$ \\
\hline M stage & 0.042 & 2.316 & $2.132-2.516$ & $<.001$ & 0.100 & 0.855 & $0.703-1.040$ & 0.117 \\
\hline No. of retrieved lymph node & 0.042 & 0.766 & $0.705-0.831$ & $<.001$ & 0.048 & 0.713 & $0.648-0.783$ & $<.001$ \\
\hline Resection type & 0.055 & 4.022 & $3.612-4.479$ & $<.001$ & 0.061 & 2.251 & $1.997-2.538$ & $<0.01$ \\
\hline Time period & 0.026 & 0.831 & $0.789-0.874$ & $<.001$ & 0.030 & 0.969 & 0.914-1.026 & 0.281 \\
\hline
\end{tabular}

A

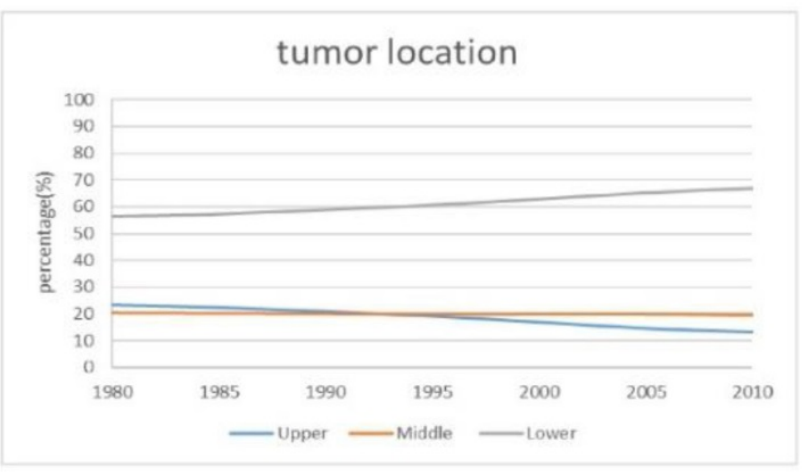

C

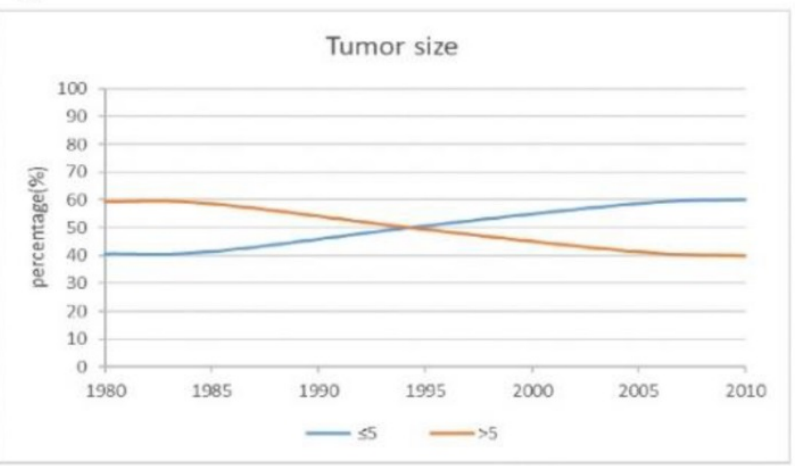

$\mathbf{E}$

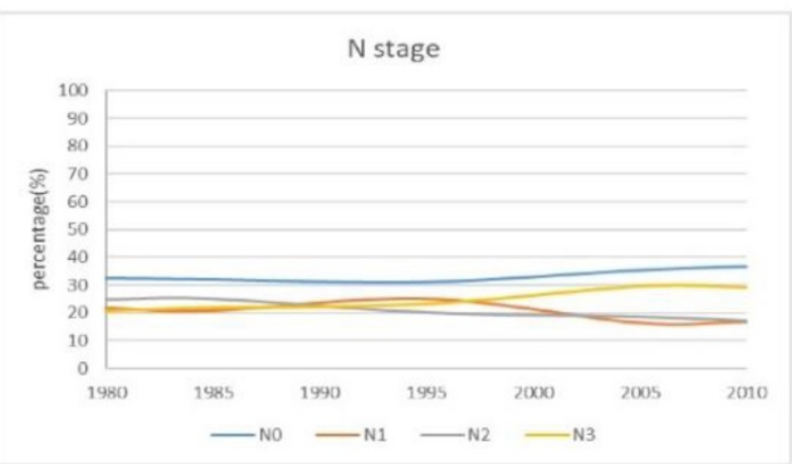

B

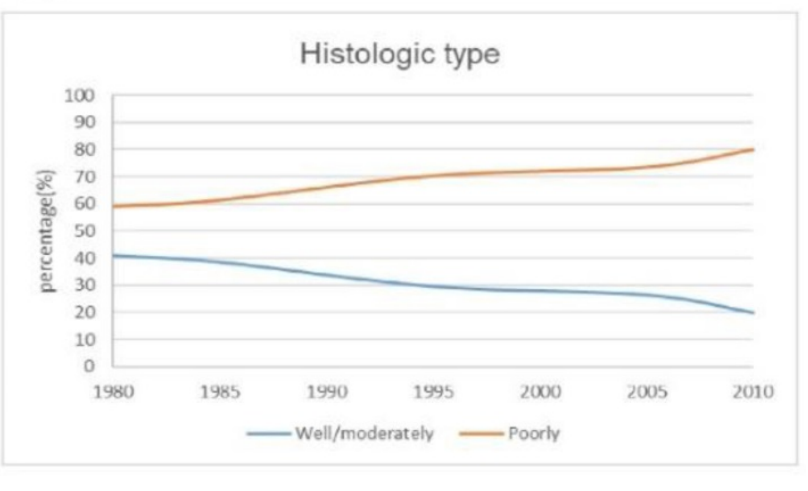

\section{D}

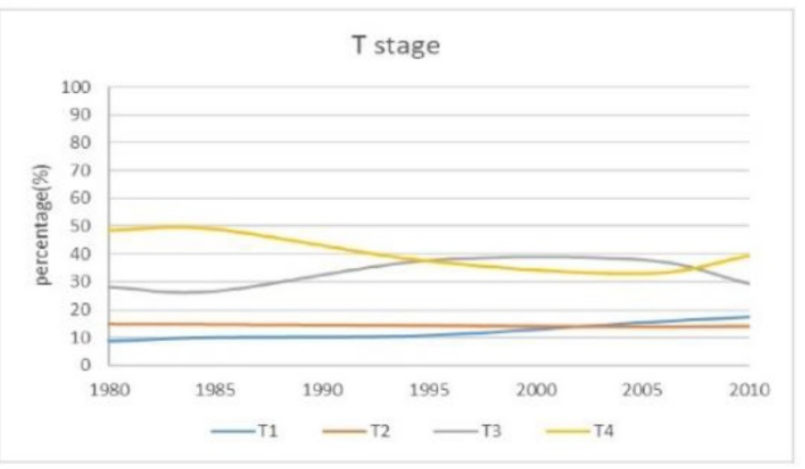

\section{$\mathbf{F}$}

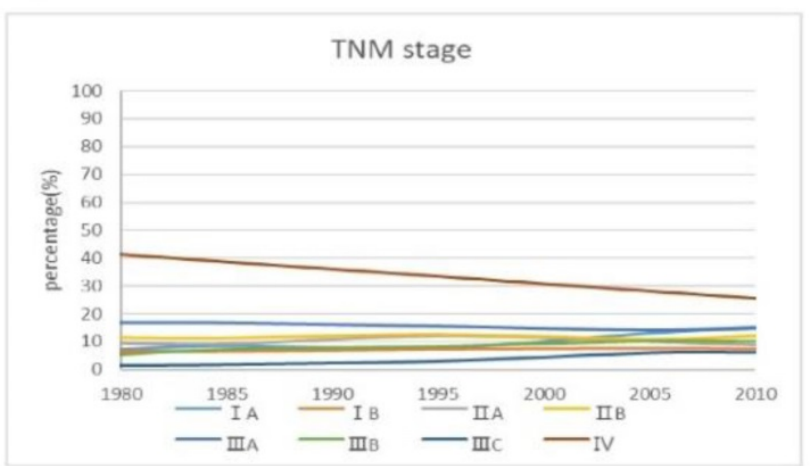

Figure 2. The curve of pathological characteristics changes over time for the patients. 
A

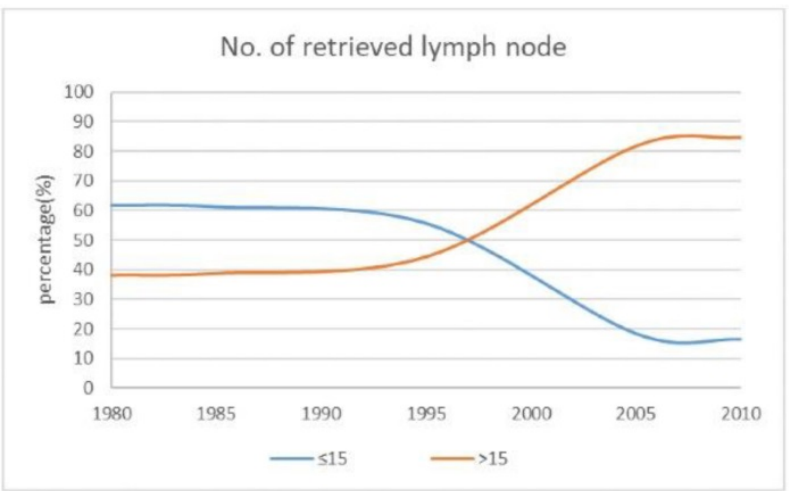

B

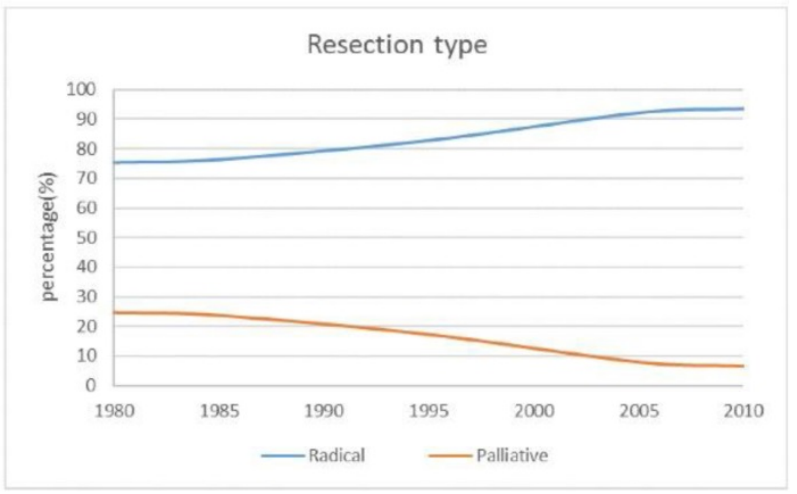

Figure 3. The curve of operative treatment changes over time for the patients.

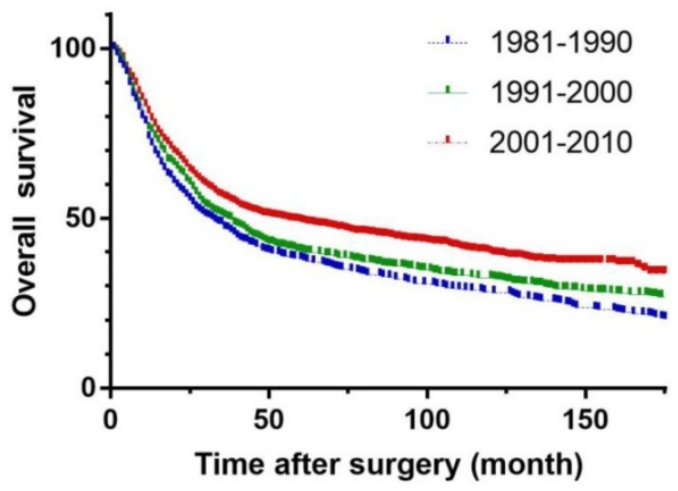

Figure 4. Overall survival of three periods in gastric carcinoma.

\section{Discussion}

In recent years, with the development of basic research, the application of radiotherapy, chemotherapy and targeted therapy, medical personnel have a greater understanding of the diagnosis and treatment of gastric cancer and have a direct or indirect effect on the effect of surgical treatment. We analyzed that the time tendency in clinicopathologic characteristics and prognosis after gastrectomy for gastric cancer over a 30-year period in our department of surgery, which is a high-volume center for gastric cancer in Northern China.

Although more young patients with gastric cancer have been reported in recent years $(12,13)$, gastric cancer still occurs predominantly in middle-aged and elderly patients. Nevertheless, the effect of age on gastric cancer remains controversial. Nakamura et al. (14) has suggested that, for EGC, youth is a protective prognostic factor. However, for advanced gastric cancer patients, young patients are prone to tumor spread and metastasis because of poor tumor differentiation, so prognosis is worse. In contrast, Saito el al. (15) debated that elderly patients undergo limited lymph node dissection and show worse prognosis, which is consistent with the results of our study.

The incidence of gastric cardia carcinoma has gradually improved in western countries (16). However, the proportion of upper gastric tumors is still small, up to $12.3 \%$ in recent time. Our analysis showed that the tumor location wasn't significant in univariate analysis, which is inconsistent with some previous studies $(17,18)$. The latter suggested that cardia cancer was an independent prognostic factor, because it's frequently undifferentiated or poorly differentiated.

Park el al (19) reported that histologic types were not significantly associated with survival in patients with stage I-III in contrast to stage IV cancer. In our study, there was an increasing trend for poorly differentiated tumor, and the proportion of well/moderately differentiated tumor gradually decreased, which is similar to the research of Wang et al (10). However, our date showed that the significance of histologic types was not obvious in single factor analysis, and was not an independent prognostic factor.

In accordance with previous authors, we conclude that the trend is for an increased incidence in small tumors compared with large tumors $(6,10)$. Adachi and Won $(20,21)$ reported that tumor size as an independent factor was connected with the prognosis of gastric cancer, which is in line with our results. $\mathrm{Zu}$ el al (22) stated that large tumors were more prone to metastasize than small tumors were, which might explain why large tumors spread more easily via direct invasion or the lymphatics.

As we all know, EGC has better prognosis compared with advanced gastric cancer $(23,24)$. As a result of popularization of gastroscopy, the proportion of gastric cancer in Japan is almost $40 \%-$ $60 \%$, which is far higher than in other countries $(20$, $21)$. We found that the incidence of EGC gradually increased in recent years despite removing the patients undergoing endoscopic resection. With 
regard to TNM stage, the general viewpoint is that the prevalence of locally advanced and metastatic tumors, especially stage IV, has decreased in contrast to the prevalence of EGC $(7,8)$. TNM stage of gastric cancer is an important basis for the selection of clinical treatment strategy and the prognostic judgement in clinical practice. Although the proportion of patients with advanced gastric cancer is decreasing, the total number of patients is still large, and they need more systematic and effective treatment to achieve better prognosis.

The eighth edition of the AJCC UICC/TNM staging system once again emphasizes the criteria of dissection for at least 16 lymph nodes, but for more accurate evaluation, the number of lymph nodes retrieved should be $>30$. Okajima et al (25) reported that the number of retrieved lymph nodes had a significant effect on survival of patients with stage II and III. In our study, the number of retrieved lymph nodes and resection type were both independent prognostic factors. Radical resection, as a surgical therapeutic tool, is the most effective way to improve the long-term survival rate of gastric cancer. However, many patients with gastric cancer are in the advanced stage during treatment. In the past few years, the deepened understandings of gastric cancer and progress in surgical techniques have increased the proportion of patients undergoing radical resection, especially those with stage II or III cancer.

The 5-year survival rate in period 3 was better than in periods 1 and 2 in our study, and the time period was statistically significant in univariate analysis but not an independent prognostic factor. These improvements may be correlated with new concepts of gastric cancer treatment, such as canonical, reasonable scope of radical cure, enhancement of comprehensive treatment and individualized treatment, provide new treatment options for patients with gastric cancer. Another new option is the use of neoadjuvant therapy, and a growing number of large clinical trials have demonstrated that radiotherapy, chemotherapy and targeted drugs can help reduce tumor recurrence and improve long-term survival rate (26-28).

The prevalence of clinicopathologic factors, such as radical and palliative resection, has transformed during the 30 years in our department. Overall survival of patients with gastric cancer has significantly improved because of earlier diagnosis and standardized surgical treatment. It is widely believed that gastric cancer has multiple causes, and its prognosis is influenced by many factors, including the environment. Early detection, diagnosis, and treatment are the key to prolonging survival of gastric cancer patients. More people are being added to the early cancer screening program to initiate treatment as early as possible and improve survival time and quality of life.

\section{Acknowledgement}

This work was supported by National Natural Science Foundation of China (No 81772549, 81572334). Authors' contributions HMX designed the research. YET took part in designing the research, collected the data, analyzed the date and wrote the manuscript. PLW collected the data, analyzed the date. SCY solved the disagreements between YET and PLW. SCY, WBH and $\mathrm{CZ}$ took part in collected the data. All the authors approved the final manuscript.

\section{Competing Interests}

The authors have declared that no competing interest exists.

\section{References}

1. Bray F, Ferlay J, Soerjomataram I, et al. Global cancer statistics 2018: GLOBOCAN estimates of incidence and mortality worldwide for 36 cancers in 185 countries. CA Cancer J Clin. 2018;686:394-424.

2. Chen W, Zheng R, Baade PD, et al. Cancer statistics in China, 2015. CA Cancer J Clin. 2016;662:115-32.

3. Zheng R, Zeng H, Zhang S, et al. Estimates of cancer incidence and mortality in China, 2013. Chin J Cancer. 2017;361:66.

4. Wang P, Sun Z, Wang W, et al. Conditional survival of patients with gastric cancer who undergo curative resection: A multi-institutional analysis in China. 2018;1245:916-24.

5. Tong JH, Sun Z, Wang ZN, et al. Early gastric cancer with signet-ring cell histologic type: risk factors of lymph node metastasis and indications of endoscopic surgery. Surgery. 2011;1493:356-63.

6. Maehara Y, Kakeji Y, Oda S, et al. Time trends of surgical treatment and the prognosis for Japanese patients with gastric cancer. $\mathrm{Br} \mathrm{J}$ Cancer. 2000;838:986-91.

7. Popiela T, Kulig J, Kolodziejczyk P, et al. Changing patterns of gastric carcinoma over the past two decades in a single institution: clinicopathological findings in 1557 patients. Scand J Gastroenterol. 2002;375:561-7.

8. Borch $\mathrm{K}$, Jonsson B, Tarpila E, et al. Changing pattern of histological type, location, stage and outcome of surgical treatment of gastric carcinoma. Br J Surg. 2000;875:618-26.

9. Ahn HS, Lee HJ, Yoo MW, et al. Changes in clinicopathological features and survival after gastrectomy for gastric cancer over a 20-year period. Br J Surg. 2011;982:255-60.

10. Wang W, Zheng C, Fang C, et al. Time trends of clinicopathologic features and surgical treatment for gastric cancer: Results from 2 high-volume institutions in southern China. Surgery. 2015;1586:1590-7.

11. Murata A, Okamoto K, Muramatsu K, et al. Time trend of medical economic outcomes of endoscopic submucosal dissection for gastric cancer in Japan: a national database analysis. Gastric Cancer. 2014;172:294-301.

12. Koea JB, Karpeh MS, Brennan MF. Gastric cancer in young patients: demographic, clinicopathological, and prognostic factors in 92 patients. Ann Surg Oncol. 2000;75:346-51.

13. Liu S, Feng F, Xu G, et al. Clinicopathological features and prognosis of gastric cancer in young patients. BMC Cancer. 2016;16:478.

14. Nakamura T, Yao T, Niho $\mathrm{Y}$, et al. A clinicopathological study in young patients with gastric carcinoma. J Surg Oncol. 1999;714:214-9.

15. Saito H, Osaki T, Murakami D, et al. Effect of age on prognosis in patients with gastric cancer. ANZ J Surg. 2006;766:458-61.

16. Maeda H, Okabayashi T, Nishimori I, et al. Clinicopathologic features of adenocarcinoma at the gastric cardia: is it different from distal cancer of the stomach? J Am Coll Surg. 2008;2062:306-10.

17. Shim JH, Song KY, Jeon HM, et al. Is gastric cancer different in Korea and the United States? Impact of tumor location on prognosis. Ann Surg Oncol. 2014;217:2332-9.

18. Talamonti MS, Kim SP, Yao KA, et al. Surgical outcomes of patients with gastric carcinoma: the importance of primary tumor location and microvessel invasion. Surgery. 2003;1344:720-7; discussion 7-9.

19. Park JM, Jang YJ, Kim JH, et al. Gastric cancer histology: clinicopathologic characteristics and prognostic value. J Surg Oncol. 2008;987:520-5.

20. Adachi $Y$, Oshiro T, Mori M, et al. Tumor size as a simple prognostic indicator for gastric carcinoma. Ann Surg Oncol. 1997;42:137-40. 
21. Im WJ, Kim MG, Ha TK, et al. Tumor size as a prognostic factor in gastric cancer patient. J Gastric Cancer. 2012;123:164-72.

22. $\mathrm{Zu} \mathrm{H}$, Wang $\mathrm{F}$, Ma Y, et al. Stage-stratified analysis of prognostic significance of tumor size in patients with gastric cancer. PLoS One. 2013;81:e54502.

23. Amin MB, Greene FL, Edge SB, et al. The Eighth Edition AJCC Cancer Staging Manual: Continuing to build a bridge from a population-based to a more "personalized" approach to cancer staging. CA Cancer J Clin. 2017;672:93-9.

24. Kao YC, Fang WL, Wang RF, et al. Clinicopathological differences in signet ring cell adenocarcinoma between early and advanced gastric cancer. 2019;222:255-63.

25. Okajima $W$, Komatsu S, Ichikawa D, et al. Prognostic impact of the number of retrieved lymph nodes in patients with gastric cancer. J Gastroenterol Hepatol. 2016;319:1566-71.

26. Bang YJ, Kim YW, Yang HK, et al. Adjuvant capecitabine and oxaliplatin for gastric cancer after D2 gastrectomy (CLASSIC): a phase 3 open-label, randomised controlled trial. Lancet. 2012;3799813:315-21.

27. Cunningham D, Allum WH, Stenning SP, et al. Perioperative chemotherapy versus surgery alone for resectable gastroesophageal cancer. N Engl J Med. 2006;3551:11-20.

28. Bang YJ, Van Cutsem E, Feyereislova A, et al. Trastuzumab in combination with chemotherapy versus chemotherapy alone for treatment of HER2-positive advanced gastric or gastro-oesophageal junction cancer (ToGA): a phase 3, open-label, randomised controlled trial. Lancet. 2010;3769742:687-97. 